\title{
Incomplete clonal deletion as prerequisite for tissue-specific minor antigen tolerization
}

\author{
Nina Pilat, ${ }^{1}$ Benedikt Mahr, ${ }^{1}$ Lukas Unger, ${ }^{1}$ Karin Hock, ${ }^{1}$ Christoph Schwarz, ${ }^{1}$ Andreas M. Farkas, ${ }^{1}$ \\ Ulrike Baranyi, ${ }^{1}$ Fritz Wrba, ${ }^{2}$ and Thomas Wekerle ${ }^{1}$ \\ 'Section of Transplantation Immunology, Department of Surgery, and 'Institute of Clinical Pathology, \\ Medical University of Vienna, Vienna, Austria.
}

\begin{abstract}
Central clonal deletion has been considered the critical factor responsible for the robust state of tolerance achieved by chimerism-based experimental protocols, but split-tolerance models and the clinical experience are calling this assumption into question. Although clone-size reduction through deletion has been shown to be universally required for achieving allotolerance, it remains undetermined whether it is sufficient by itself. Therapeutic Treg treatment induces chimerism and tolerance in a stringent murine BM transplantation model devoid of myelosuppressive recipient treatment. In contrast to irradiation chimeras, chronic rejection (CR) of skin and heart allografts in Treg chimeras was permanently prevented, even in the absence of complete clonal deletion of donor MHC-reactive T cells. We show that minor histocompatibility antigen mismatches account for CR in irradiation chimeras without global T cell depletion. Furthermore, we show that Treg therapy-induced tolerance prevents CR in a linked suppression-like fashion, which is maintained by active regulatory mechanisms involving recruitment of thymus-derived Tregs to the graft. These data suggest that highly efficient intrathymic and peripheral deletion of donor-reactive $T$ cells for specificities expressed on hematopoietic cells preclude the expansion of donor-specific Tregs and, hence, do not allow for spreading of tolerance to minor specificities that are not expressed by donor BM.
\end{abstract}

Conflict of interest: The authors have declared that no conflict of interest exists.

Submitted: December 8, 2015 Accepted: April 19, 2016 Published: May 19, 2016

Reference information: JCI Insight. 2016;1(7):e85911 doi:10.1172/jci.insight.85911.

\section{Introduction}

Induction of donor-specific tolerance, defined as a state of donor-specific unresponsiveness without a need for ongoing pharmacologic immunosuppression, remains an elusive goal in clinical organ transplantation. Tolerance is generally considered to be an active process that mechanistically occurs at 2 different levels, centrally by intrathymic deletion of developing $\mathrm{T}$ cells and peripherally by both deletional and nondeletional mechanisms. Although tolerance has been achieved and studied in numerous laboratory animal models, translation into the human setting failed in all but one case, namely the creation of hematopoietic chimeras via BM transplantation (BMT). The exceptional clinical potential of the mixed chimerism approach in the induction of donor-specific immunological tolerance has been underscored by a number of clinical trials in renal transplant recipients (1-3). Widespread clinical application, however, is hindered not only by the toxicity of the required cytoreductive recipient conditioning and the risk of graft-versushost disease (GVHD) (4), but also by the relative lack of efficacy of current protocols achieving durable tolerance in only $40 \%-63 \%$ of haplo-matched patients (5). Central tolerance with intrathymic deletion of donor-reactive $\mathrm{T}$ cell clones is thought to be the hallmark and key to success of the mixed chimerism approach (6-9), distinguishing its potency from other tolerance approaches that all failed when translated into the human setting (10). However, the mechanisms leading to indefinite allograft survival despite the loss of chimerism in some patients still have to be elucidated $(1,4)$. Moreover, preclinical data demonstrated that current renal tolerance protocols are likely to fail to induce tolerance toward cardiac or lung allografts and improved protocols will be needed to achieve tolerance in heart or lung recipients (11).

$\mathrm{CD}^{+}{ }^{+} \mathrm{CD} 25^{+} \mathrm{FoxP}^{+}$Tregs are key mediators of self-tolerance, and therapeutic Treg cell treatment by adoptive transfer has been highly effective in murine models to treat autoimmune diseases and GVHD (12). Although Tregs have been shown to be a potent therapeutic target in the prevention of allograft rejection, they do not induce skin graft tolerance across full MHC barriers on their own $(13,14)$. However, Treg 
A
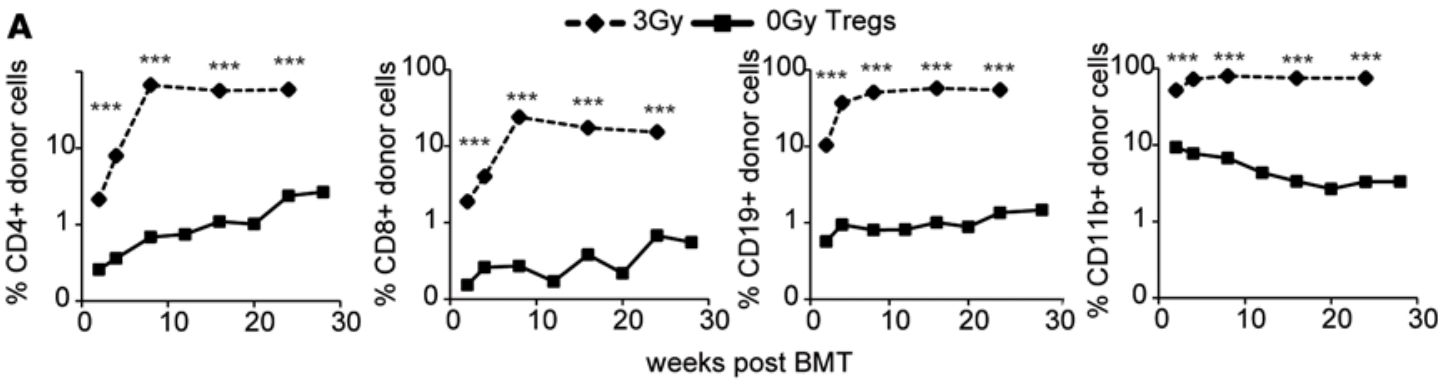

$$
\text { B }
$$
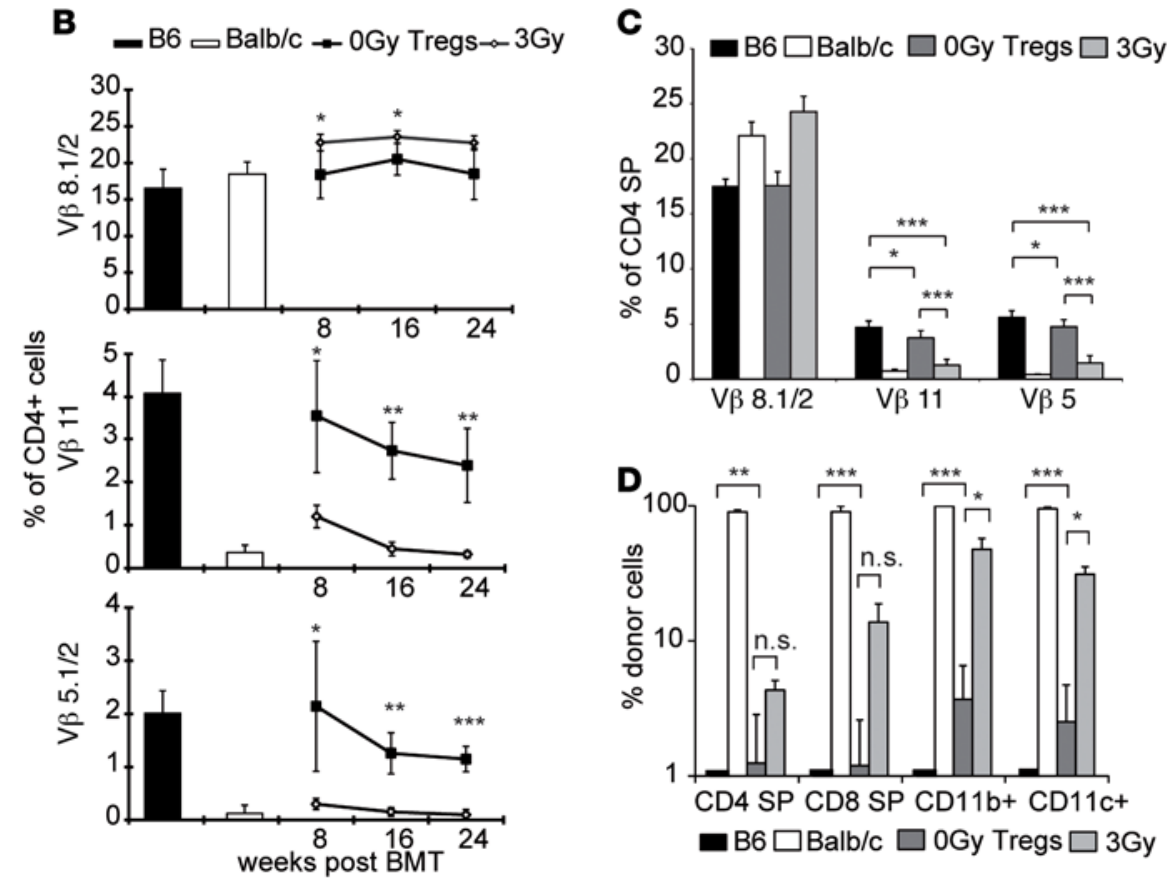

E Treg-chimera
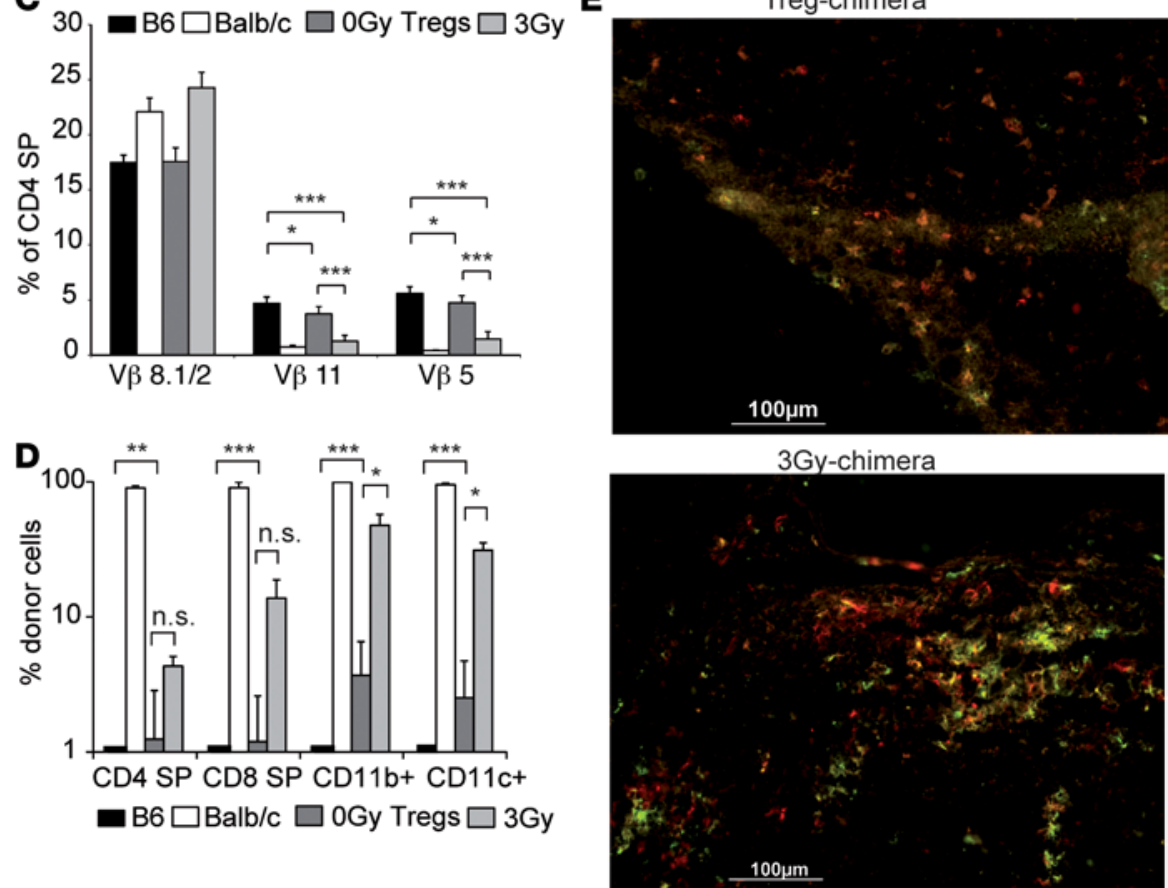

Figure 1. Treg treatment replaces irradiation to induce multilineage chimerism and central tolerance. Groups of B6 mice were treated with a costimulation blockade-based BMT protocol and grafted with $2 \times 10^{7}$ BALB/c BM cells to induce donor-specific tolerance. BMT recipients were additionally treated with nonmyeloablative ( $3 G y, n=8$ ) irradiation or recipient-derived natural Tregs ( $n$ Tregs) + (0-Gy Tregs, $n=6)$. (A) The majority of recipients in both groups developed permanent multilineage chimerism. Donor $\left(\mathrm{H}-2 \mathrm{D}^{\mathrm{d}}\right)$ chimerism among leukocytes of the $\mathrm{T}$ cell (CD4 ${ }^{+}$and $\left.\mathrm{CD} 8^{+}\right)$, B cell (CD19+), and myeloid (CD11b/Mac1 ${ }^{+}$) lineage. (B) Deletion of donor-reactive T cells (correlates to V $\beta 11$ and V $\beta 5$ expression) was assessed by flow cytometry of peripheral blood at multiple time points after BMT. (C) Central tolerance was assessed by $\mathrm{V} \beta$ expression pattern on intrathymic single-positive (SP) CD4 T cells and (D) the presence of intrathymic chimerism 30 weeks after BMT. Flow cytometric staining verified the engraftment of antigen-presenting donor cells in the thymus and central deletion (30 weeks after BMT). (E) Immunofluorescent staining of frozen thymic sections showed coexpression of CD11C (red) and donor MHC II (I-Ek/d, green). Shown as mean percentage + SD; results are representative of at least 3 independent experiments; ${ }^{* *} P<0.0005,{ }^{*} P<0.005,{ }^{*} P<0.05 ; 2$-tailed $t$ test.

therapy has been shown to act synergistically with BMT in the induction of transplantation tolerance by us and others $(14,15)$. Previously, we developed a BMT protocol in which therapeutic Treg infusion led to engraftment of allogeneic BM without cytoreductive recipient conditioning, thereby obviating toxicity (15). The tolerance achieved was superior to well-established nonmyeloablative BMT protocols (16) that rely on a 3-Gy dose of total body irradiation (TBI) (17). However, the mechanisms of tolerance in Treg chimeras responsible for the superior efficacy of this protocol over irradiation chimeras remain to be determined.

\section{Results}

Treg treatment induces full immunological tolerance despite incomplete central and peripheral deletion. $\mathrm{B} 6\left(\mathrm{H}-2^{\mathrm{b}}\right)$ recipient mice were grafted with fully mismatched BALB/c $\left(\mathrm{H}-2^{\mathrm{d}}\right) \mathrm{BM}$ cells under the cover of short-term costimulation blockade and received either Tregs without irradiation ("Treg protocol") (15) or a well-established protocol employing nonmyeloablative TBI (3 Gy) (16). Both protocols led to stable, multilineage chimerism in the majority of mice (Treg 24/27, 3 Gy 29/33; $P=1.000$; data not shown) with irradiation chimeras developing significantly higher levels of chimerism in all tested lineages in peripheral blood (Figure 1A) and lymphoid 
A

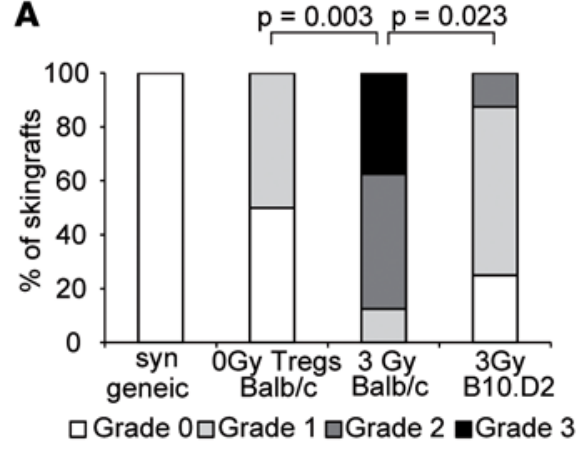

C Balb/c

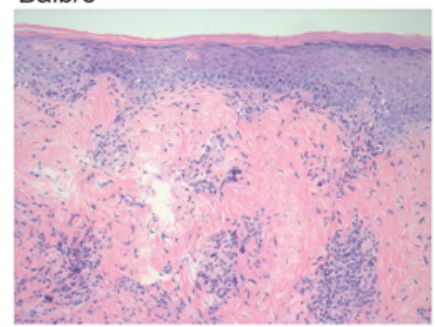

B

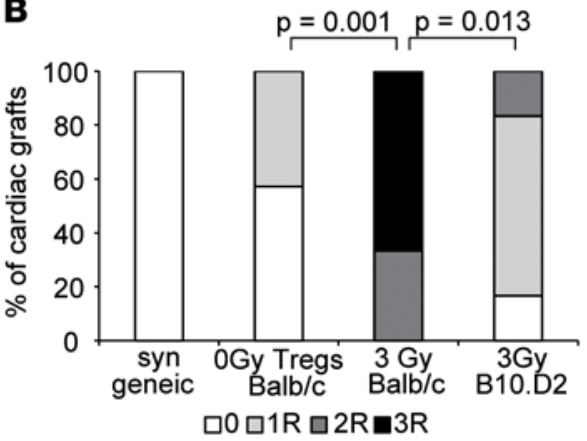

B10.D2

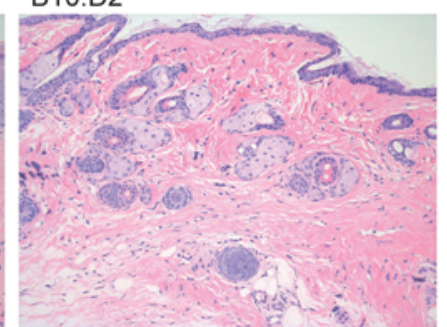

Figure 2. Treg but not 3 Gy irradiation treatment induces full immunological tolerance toward non-MHC antigens in mixed chimeras. (A and $\mathbf{B})$ Donor-specific tolerance was assessed by grafting of donor-type fully mismatched (BALB/C) or MHC-mismatched (B10.D2) skin and heart 4-6 weeks after BMT. Classification of (A) skin allograft pathology and (B) heart allograft rejection scores for fully mismatched (3 Gy, $n=6$; Tregs, $n=7$ ) and MHC-mismatched grafts ( $3 \mathrm{~Gy}, n=6$ ) are shown. Fisher's exact test was used to compare rejection scores between groups. Data are representative of at least 2 independent experiments. (C) Representative histology from 3-Gy skin grafts ( $>120$ days after skin grafting; H\&E staining magnification $\times 200$ ) for fully mismatched grafts (Balb/c, left) and MHC mismatched grafts (B10.D2, right; $n=6$ / group) is shown.

organs (30 weeks after BMT, data not shown, consistent with previous data; ref. 18). Although chimerism in Treg chimeras was rather low, it was of multilineage nature and remained stable throughout followup. Deletion of donor-reactive $\mathrm{T}$ cell clones was measured by following $\mathrm{T}$ cells expressing particular TCR V $\beta$ subunits (i.e., $\mathrm{V} \beta 11^{+}$and $\mathrm{V} \beta 5^{+} \mathrm{T}$ cells recognizing superantigens presented by donor $\mathrm{MHCII} \mathrm{I}-\mathrm{E}$, which is not expressed in B6 mice; $\mathrm{V} \beta 8^{+}$expression was used as unspecific control) (15). Deletion in 3-Gy chimeras started immediately after BMT, was significant as early as 4 weeks after BMT (Supplemental Figure 1; supplemental material available online with this article; doi:10.1172/jci.insight.85911DS1), and became complete around 16 weeks after BMT in peripheral blood (Figure 1B). In Treg chimeras, although evident, deletion was significantly less pronounced throughout followup. Investigation of intrathymic $\mathrm{V} \beta$ expression patterns revealed central deletion among single-positive CD4 thymocytes in Treg chimeras, although of limited extent, whereas central deletion in 3-Gy chimeras was almost complete (Figure 1C). Intrathymic chimerism was evident in T cells, myeloid-derived cells, and DCs (Figure 1D), confirming the presence of donor-derived DCs in 3-Gy and also Treg chimeras (Figure 1E). Interestingly, although 3-Gy chimeras demonstrated high levels of chimerism and almost complete central and peripheral deletion of donor-reactive $T$ cells, they failed to induce full immunological tolerance and prevent chronic rejection (CR) in fully mismatched skin and heart allografts (17) as demonstrated previously.

Treg treatment prevents minor antigen-mediated rejection. Therapeutic Treg treatment is suggested to directly contribute to tolerance by regulatory mechanisms $(14,15,17)$, and we hypothesized that tissue-specific minor histocompatibility antigens ( $\mathrm{miHa}$ ) account for $\mathrm{CR}$ in irradiation-induced chimeras, as they are not presented by recipient medullary thymic epithelial cells (mTECs) (19). To directly test the effect of minor antigen mismatches, B10.D2 $\left(\mathrm{H}-2^{\mathrm{d}}\right)$ mice, which lack miHa mismatches to B6 recipient strain, were utilized. Donor grafts (Balb/c and B10.D2) survived long term (> 150 days) in both Treg and 3-Gy chimeras, whereas unrelated third-party $(\mathrm{C} 3 \mathrm{H})$ grafts were rapidly rejected (data not shown). Interestingly, irradiation chimeras show CR in the fully mismatched but not MHC-mismatch combination (Figure 2, A and B), while Treg chimeras showed full immunological tolerance toward fully MHC- and miHa-mismatched skin (Figure 2A) and cardiac allografts (Figure 2B). In 3-Gy chimeras, long-term surviving grafts from fully mismatched but not MHC-mismatched grafts present with leukocyte infiltrates and substantial tissue damage (Figure 2C). These data suggest that chimeras induced by nonmyeloablative irradiation and costimulation blockade are incompletely tolerant toward non-MHC donor antigens.

Tolerance is maintained by regulatory mechanisms involving infectious tolerance. In light of these data, we wanted to know whether the excellent long-term graft survival in recipients of the Treg protocol is indeed rather mediated by regulatory than by deletional mechanisms. Long-term tolerant chimeras ( $>100$ days) were challenged with anti-CD25 and anti-CTLA4, which is critically required for Treg function (20), or anti-PD1, which was shown to be required for Treg induction and maintenance (21). Administration 
A

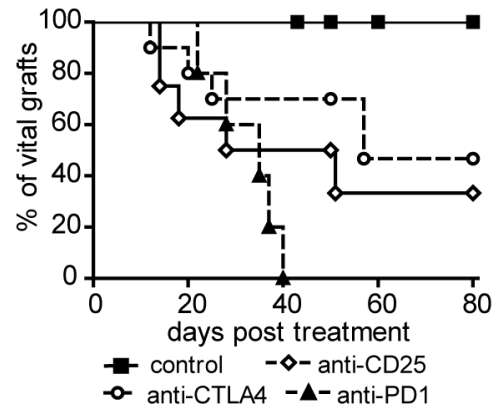

D

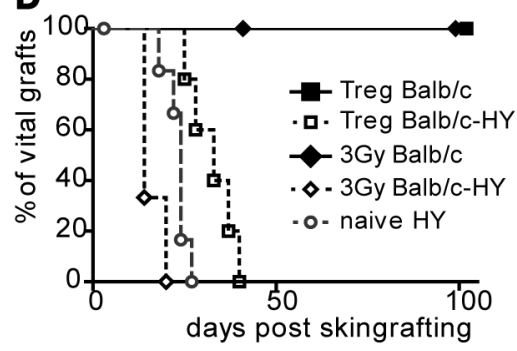

B
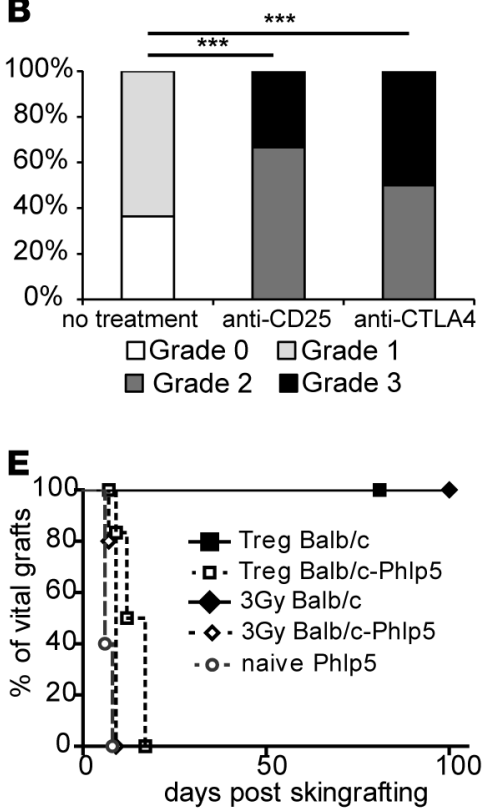

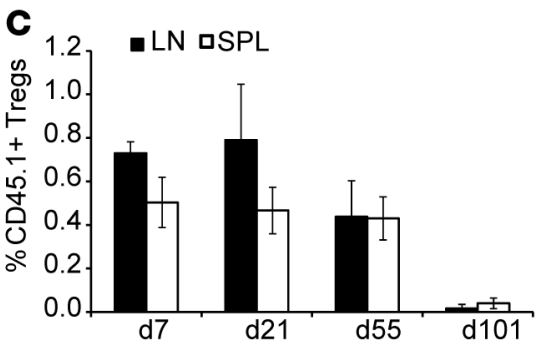

F -3Gy chimera aTreg chimera

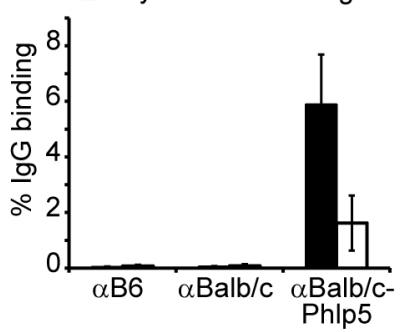

Figure 3. Treg-treated mice maintain tolerance through regulatory mechanisms and confer infectious tolerance. (A) Long-term tolerant mice were challenged with anti-CD25 mAb, anti-CTLA4 mAb, or anti-PD1 mAb 100 days after BMT to assess the impact of Tregs in long-term graft survival (control $[n=11]$ vs. anti-CD25 [ $n=7], P=0.0025$; vs. anti-CTLA4 [ $n=10], P=0.0158$; vs. anti-PD1 [ $n=5], P<0.0001$; log-rank). Data are pooled from 2 independent experiments. (B) Surviving grafts of Treg chimeras were analyzed for signs of chronic rejection (CR; $>50$ days after treatment; ${ }^{* * *} P>0.0001$, Fisher's exact test). (C) The longevity of therapeutically transferred (CD45.1 congenic) Tregs ( $n=3 /$ time point) was followed by flow cytometry in lymph nodes (LN) and spleen (SPL). ( $\mathbf{D}$ and E) Chimeric mice conditioned with either 3-Gy or Treg-BMT protocols are challenged with skin grafts for tolerance assessment and infectious tolerance. (D) Mice received fully mismatched donor grafts (BALB/c) and donor grafts including an additional minor antigen mismatch (BALB/cHY). Survival is shown for 3-Gy chimeras (3-Gy BALB/c, median survival time [MST] > 100 days, $n=5 ; 3-G y$ BALB/c-HY, MST = 14 days, $n=3$ ), Treg chimeras (Treg BALB/c, MST > 100 days, $n=10$; Treg BALB/c-HY, MST = 33 days, $n=5$ ), and naive mice (naive HY, MST = 24 days, $n=7$ ). (E) Rejection kinetics for Phlp5 allergen as additional minor antigen are shown (3-Gy BALB/c, MST > 100 days, $n=5 ; 3-$ Gy BALB/c-PhIp5, MST = 9 days, $n=5$; Treg BALB/c, MST $>80$ days, $n=10$; Treg BALB/c-PhIp5, MST = 14.5 days, $n=7$; naive BALB/c-Phlp5, MST = 6 days, $n=5)$. Treg chimeras show prolonged survival vs. $3-G y$ chimeras ( $P=0.0046$ for $\mathrm{HY} ; P=0.0118$ for Phlp5) or naive mice $(P=0.0051$ for $\mathrm{HY} ; P=0.0021 \mathrm{for}$ Phlp5; all using log-rank). (F) The reactivity of sera ( 3 weeks after skin graft rejection) from 3-Gy chimeras $(n=5)$ and Treg chimeras $(n=6)$ with syngeneic (B6), donor (BALB/c), and BALB/c-PhIp5 thymocytes is shown by flow cytometry through indirect staining with anti-mouse IgG.

of anti-CD25 or anti-CTLA4 led to rejection of skin grafts in $\sim 50 \%$ of Treg chimeras (but not irradiation chimeras, which is in line with previous data; refs. 22 and 23), with significant histological signs of rejection in remaining grafts (Figure $3, \mathrm{~A}$ and $\mathrm{B}$ ). Interestingly, anti-PD1 uniformly led to rejection of allografts in Treg chimeras, underlining the importance of PD1 in peripheral tolerance (refs. 24, 25, and Figure 3A). Challenge of 3-Gy chimeras with either compound did not induce rejection of skin grafts in any group, and histological examination did not reveal statistical differences between groups (Supplemental Figure 2). Notably, hematopoietic chimerism remained stable in all mice (data not shown), suggesting that anti-CD25/anti-CTLA4/anti-PD1 trigger immunity toward tissue-specific antigens not expressed on hematopoietic cells but do not trigger immunity directed toward donor hematopoietic cells. Lack of anti-donor antibodies proposes cell-mediated rejection without humoral anti-donor response or humoral response against non-MHC antigens (tissue-specific miHa) only. These data strongly suggest that regulatory mechanisms maintain tolerance toward tissue-specific miHa of the donor in Treg chimeras. Importantly, therapeutically transferred Tregs vanish over time (Figure 3C) and are therefore not directly mediating long-term graft survival $(15,23)$.

To test whether a linked suppression-like mechanism is set in motion and contributes to regulation, which was also proposed in the clinical setting of transient chimerism (26), tolerant mice received a second skin graft with an additional single-antigen mismatch from male BALB/c (Figure 3D) or BALB/c-Phlp5 (BALB/c mice ubiquitously expressing highly immunogenic single-antigen Phlp5) (Figure 3E) mice. Rejection was moderately, but significantly delayed in Treg chimeras in comparison with irradiation chimeras or naive mice; while noteworthy, none of the mice experienced rejection of primary donor skin graft. General immunosuppression or immunoincompetence in either BMT model was precluded by third-party grafts, which were rapidly rejected in all mice (data not shown). Importantly, upon rejection, chimeras developed 
A

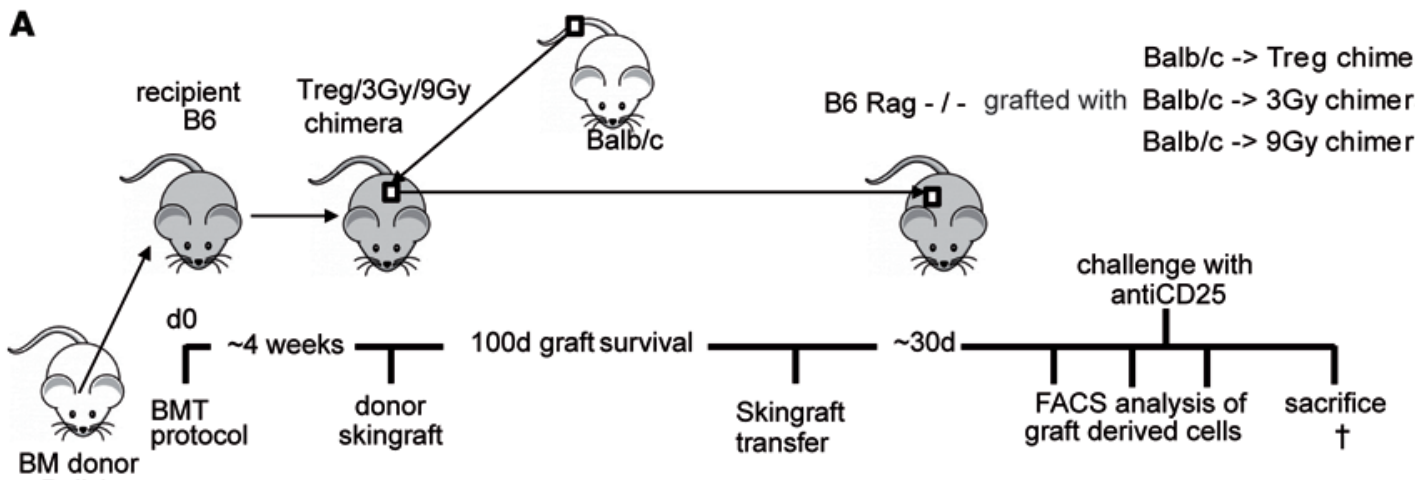
Balb/c

B
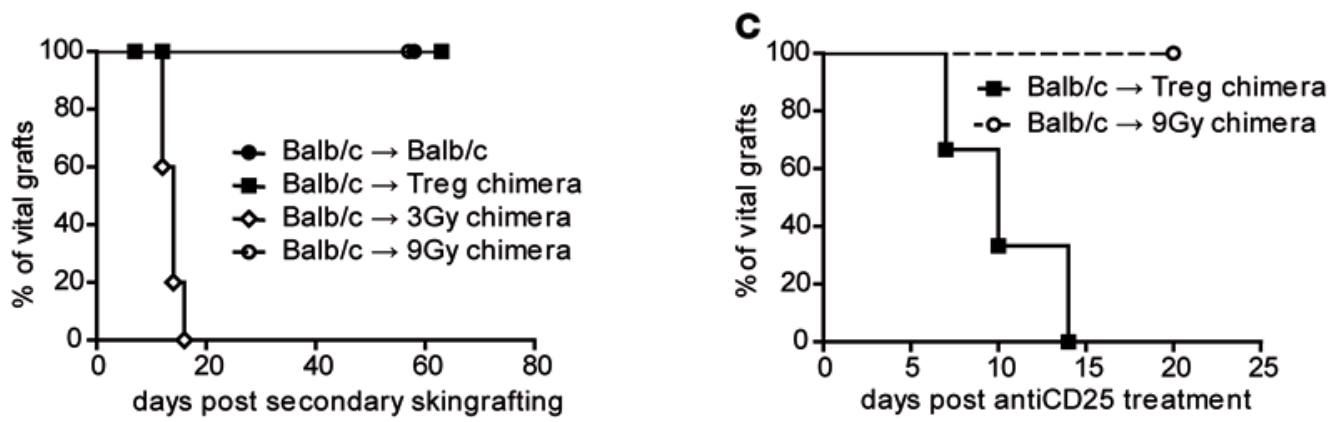

D Balb/c -> Treg chimera

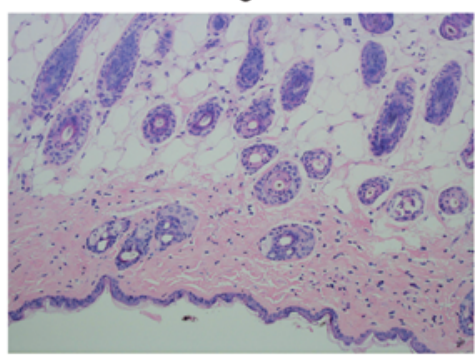

$\mathbf{F}$
Balb/c -> 9Gy chimera

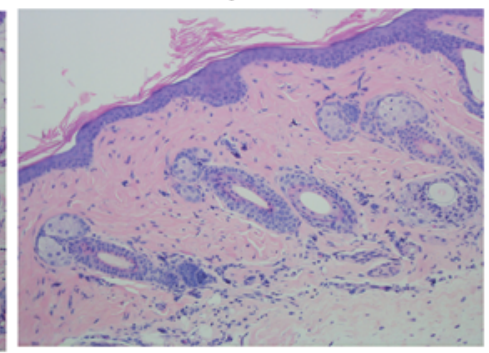

control
E

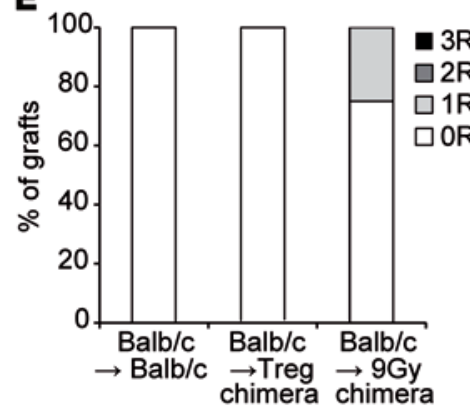

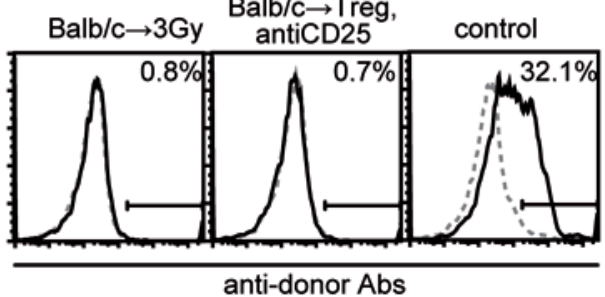

Figure 4. Tolerance in Treg chimeras is maintained by active intragraft regulatory mechanisms. (A) Schematic illustration of skin transfer experiment setup. (B) Fully mismatched Balb/c allografts from long-term (100 days) tolerant recipients from different treatment protocols are transferred onto RAG $\sigma^{-1-}$-recipient mice. Legend depicts primary donor $\rightarrow$ recipient combination (BALB/c $\rightarrow$ BALB/c, $n=3 ; \rightarrow$ Treg chimera, $n=7 ; \rightarrow 3$-Gy chimera, $n=5 ; \rightarrow 9$-Gy chimera, $n=5$ ). (C) Selected RAG ${ }^{-1-}$ recipients with secondary skin grafts are challenged with anti-CD25 (40 days after skin graft transfer; $n=3$ each group). (D) Representative histology from transferred skin grafts ( $>8$ weeks postsecondary transfer; H\&E staining magnification $\times 200)$. (E) Classification of skin allograft pathology $(>8$ weeks after transfer, $n=3$ per group). (F) Reactivity of sera (>8 weeks after skin grafting) with syngeneic (B6; dotted gray) and donor (BALB/c; black) thymocytes shown by flow cytometry through indirect staining with anti-mouse IgG. Representative histograms are shown for RAC skin graft recipients from the groups: 3-Gy chimeras, Treg chimeras after anti-CD25 treatment, and naive control after skin graft rejection. ${ }^{\dagger}$, time of sacrifice ( d60).

antibodies specific for BALB/c-Phlp5 (Figure 3F). Levels of Phlp5-specific antibodies in 3-Gy chimeras were similar to naive mice (data not shown), whereas humoral response in Treg chimeras was less pronounced. Therefore, we suggest that regulatory mechanisms reminiscent of linked suppression appear in Treg chimeras that might contribute to the acceptance of donor grafts in the absence of complete central deletion of donor-reactive $\mathrm{T}$ cells by downmodulating immunity toward tissue-specific miHa donor antigens.

Tolerance in Treg chimeras is actively maintained in the graft by recruitment of thymus-derived Tregs. As it is the tissue-specific miHA that need to be tolerized by regulatory mechanisms, we hypothesized that intragraft 
A

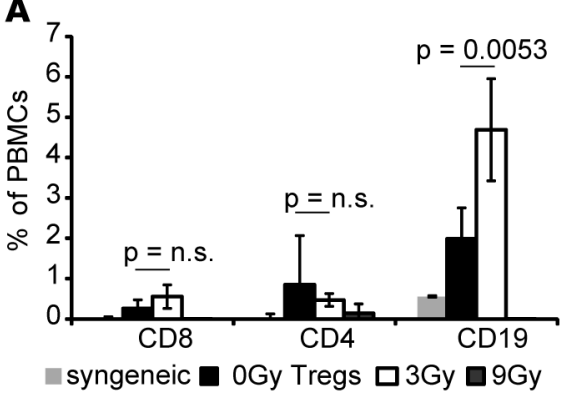

C

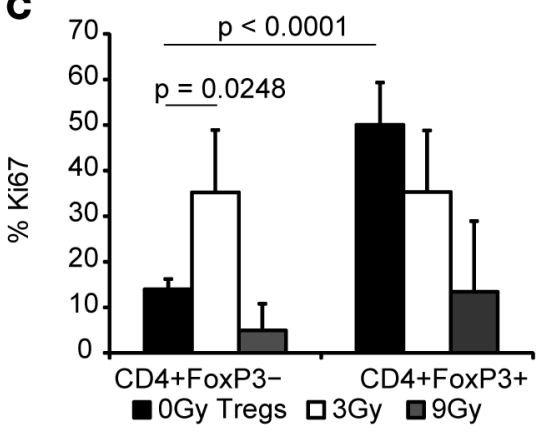

B

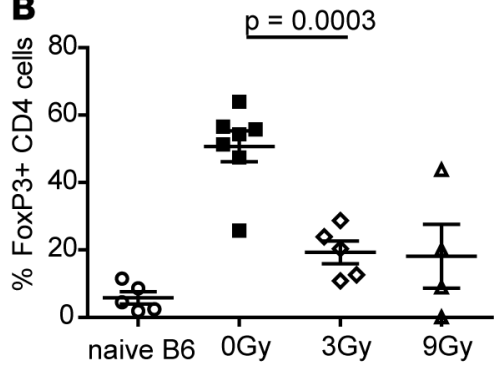

D

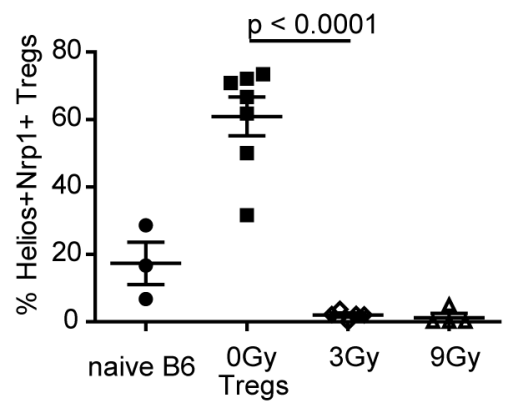

Figure 5. Thymus-derived Tregs are recruited into the graft and prevent rejection of non-MHC antigens. (A) Peripheral blood of secondary graft recipients (syngeneic $n=3$; 0-Gy Tregs, $n=7$; 3 Gy, $n=5$; $9 G y, n=5)$ was analyzed for graft-derived CD4, CD8, and CD19 populations and (B) FoxP3 ${ }^{+}$ cells among $\mathrm{CD} 4^{+}$populations (naive $\mathrm{B} 6$ were used as control). (C) Proliferation was measured by Ki67 expression in Treg (FoxP3+) and non-Treg (FoxP3-) CD4 populations. (D) Thymic origin of graft-derived Tregs was determined by Helios and Neuropilin-1 (Nrp1) coexpression in $\mathrm{CD}^{+} \mathrm{FoxP3}^{+}$ cells (mean percentages + SD of indicated cell populations are shown; 2-tailed $t$ test).

Tregs are critical. We therefore regrafted skin from long-term tolerant mice to RAG-deficient recipients in order to examine intragraft $\mathrm{T}$ and $\mathrm{B}$ cell populations and the role of graft-derived Tregs (Figure 4A). Grafts were obtained from mice receiving Treg-BMT or 2 different irradiation-based BMT protocols, either nonmyeloablative (3 Gy; mixed chimeras) or myeloablative (9 Gy; full chimeras) TBI. All grafts from 3-Gy chimeras were rejected (Figure 4B), presumably due to homeostatic proliferation of donor miHa-reactive $\mathrm{T}$ cell clones emerging from the graft. Grafts derived from Treg chimeras were accepted indefinitely, as were those of 9-Gy chimeras. Treg depletion by anti-CD25 abrogated graft acceptance in Treg but not 9-Gy chimeras (Figure 4C), demonstrating that intragraft Tregs actively prevent rejection in recipients of the Treg protocol. Long-term tolerant grafts from Treg chimeras were devoid of structural changes due to $\mathrm{CR}$ but presented with leukocyte infiltrates, including FoxP3 ${ }^{+}$-expressing cells (15). Importantly, histological examination of secondary grafts (60 days after regrafting) showed that leukocytes have emigrated from grafts, showing neither infiltrates nor signs of rejection in both Treg and 9-Gy chimeras, resembling syngeneic controls (Figure 4, D and E). None of the recipients developed anti-donor (MHC) antibodies, although B cell reconstitution in mice receiving grafts from 3-Gy chimeras was substantial (Figure 4F).

Flow cytometric analysis (FCM analysis) of peripheral blood 30 days after skin graft transfer revealed that lymphopenic hosts were repopulated with substantial numbers of graft-derived, recipient-type $\mathrm{T}$ and B cells in groups that received grafts from Treg or 3-Gy chimeras but not 9-Gy chimeras or syngeneic grafts (Figure 5A). With regard to chimerism, donor hematopoietic cells were absent in all groups (data not shown), likely due to NK-mediated elimination of donor cells. Mice that received grafts from Treg or 3-Gy chimeras showed comparable numbers of T cells; however, B cell numbers were significantly higher in the 3-Gy group. Numbers of $\mathrm{CD}^{+} \mathrm{FoxP}^{+}$Tregs among CD4 T cells were significantly higher in the Treg group, and these Tregs were highly proliferating, which is in line with in vivo skin graft survival data (Figure 4B) and provides additional evidence of active regulatory mechanisms (Figure 5, B and C). The proliferative rate of FoxP3- CD4 cells was significantly higher among the group that received 3-Gy skin grafts, indicating the intragraft presence of donor-reactive conventional $\mathrm{T}$ cells. Mice with Treg chimera-derived grafts showed a significantly increased proliferative rate among the $\mathrm{CD}^{+} \mathrm{FoxP}^{+}$population compared with the $\mathrm{CD}^{+}{ }^{+}$FoxP3 $3^{-}$population (Figure $5 \mathrm{C}$ ), suggesting TCR activation in an antigen-dependent manner (27). Furthermore, the rate of dividing conventional $\mathrm{T}$ cells was significantly decreased in the Treg group compared with 3-Gy group. Notably, the majority of $\mathrm{CD}^{+} \mathrm{FoxP}^{+}$Tregs in the Treg group (but not in irradiation groups or naive mice) expressed markers associated with thymic origin (ref. 28 and Figure 5D). These data suggest recruitment of thymus-derived Tregs to the allograft rather than induction of regulatory phenotype just in the periphery, thereby reducing the risk of reconversion to effector $\mathrm{T}$ cell phenotype. 


\section{Discussion}

In this study, we investigated the mechanisms of Treg-induced immunological tolerance in the mixed chimerism setting. Whereas nonmyeloablative irradiation leads to high levels of chimerism and almost complete deletion of donor-reactive $\mathrm{T}$ cell clones (as measured by the expression of certain superantigen-reactive TCR families), full immunological tolerance could not be achieved. Adoptive transfer of therapeutic levels of highly activated polyclonal recipient-strain Tregs not only obviates the need for myelosuppression, but also confers true and robust immunological tolerance toward the donor strain.

Whereas in 3-Gy chimeras, Tregs are dispensable and central tolerance and intrathymic deletion are sufficient in the maintenance phase of tolerance, Treg chimeras require active regulatory mechanisms and the presence of Tregs and negative costimulatory pathways for sustained tolerance. Depletion of Tregs or disruption of negative costimulatory pathways CTLA4 and PD1 leads to split tolerance, in which central tolerance prevents the rejection of donor stem cells but peripheral tolerance cannot be maintained and skin grafts are chronically rejected. However, it could be possible that donor stem cells are protected in a stem cell niche environment and less susceptible to rejection (29), which therefore prevents loss of chimerism. The lack of detectable anti-donor antibodies suggests that skin graft rejection after interference with regulatory mechanisms is either mainly cell mediated or humoral response is directed against non-MHC antigens, which are not detectable by FCM crossmatch (FCXM).

Whether or not the situation in 3-Gy chimeras can be described as split-tolerance will be a matter of debate; however, it emphasizes the importance of using stringent strain combinations in order to facilitate translation into the clinical situation (30).

Tolerance in Treg chimeras is not restricted to antigens expressed on hematopoietic cells, which is in stark contrast to 3-Gy chimeras in which miHa mismatches cause chronic rejection in a substantial fraction of chimeras. We assume that this finding is due to the low or absent expression of certain miHa antigens on hematopoietic cells, which excludes them from negative selection. Further exploration of these findings revealed that rejection of donor skin expressing an additionally introduced (nondonor) antigen is delayed in Treg chimeras but not 3-Gy chimeras, suggesting that, in Treg chimeras, regulatory mechanisms are capable of suppressing immunity toward tissue antigens not expressed on donor BM.

In light of these data, we propose that donor-specific tolerance in Treg chimeras is most likely maintained by 2 different mechanisms: firstly, therapeutic Tregs directly suppress BM rejection, which leads to chimerism and subsequent central tolerance to donor MHC, and secondly, therapeutic Treg transfer in the absence of cytotoxic danger-prone host conditioning establishes a tolerogenic state involving linked suppression-like mechanisms that protect allografts from rejection directed toward tissue-specific antigens. The persistent chimerism in 3-Gy chimeras and in Treg chimeras after breakdown of peripheral tolerance argues that there is tolerance to minor antigens on the hematopoietic cells or that there is low-level marrow rejection that is not apparent because of the self-renewal of donor stem cells.

Mechanistically, acceptance of grafts from myeloablatively conditioned (9 Gy) full chimeras indicates that myeloablation eliminates $\mathrm{T}$ cell populations that escape nonmyeloablative irradiation and remain untolerized in 3-Gy chimeras. As T cells directed against donor-MHC are efficiently deleted (peripherally and centrally) in 3-Gy chimeras (16), and as tissue-specific antigens are less prone to tolerization through deletion even in physiological self-tolerance (31), T cells with reactivity to tissue-specific donor antigens are most likely responsible for the observed rejection in 3-Gy but not 9-Gy chimeras.

Highly efficient intrathymic and peripheral deletion of donor-reactive $\mathrm{T}$ cells for specificities expressed on hematopoietic cells, as seen in TBI chimeras, presumably precludes the expansion of donor-specific Tregs and, hence, does not allow for the spreading of infectious tolerance to minor specificities (both indirect and tissue-specific) that are not strongly expressed by donor marrow and therefore do not induce deletion under cover of costimulation blockade. Lethal TBI, on the other hand, is likely to deplete all preexisting T cells, making additional peripheral tolerance mechanisms and the help of Tregs obsolete. Still, the question arises how newly developing $\mathrm{T}$ cells in these full chimeras are tolerized against tissue-specific antigens. Although we can only speculate about the detailed mechanisms, we think that differences in TCR repertoire and impaired immunocompetence in these mice contribute to excellent graft function in full chimeras (32). However, it is not a justifiable treatment for transplant patients without concurrent malignancies due to serious life-threatening risks like engraftment failure or GVHD.

Investigation of intragraft cell populations by graft transfer to immunoincompetent hosts lacking $\mathrm{T}$ and B cells revealed that, although both protocols (3 Gy and Tregs) presented with leukocyte infiltrates, the ratio 
of Tregs versus T effector cells (Teff cells) indicates a tolerant state in Treg but not 3-Gy chimeras. Despite partly unspecific proliferation in a lymphopenic environment, and an inflammatory environment due to graft-related tissue injury, the high percentage of Tregs in grafts derived from Treg chimeras ensures graft survival. In contrast, proliferation of graft-infiltrating Teff cells in 3-Gy chimeras causes rapid rejection of grafts in previously immunoincompetent hosts. We intended to use Helios and Neuropilin-1 (Nrp1) (which are considered as markers of thymic origin, refs. 33-35) to determine whether graft-infiltrating Tregs are of thymic origin or peripherally induced in the graft. While the data suggest that the majority of graft-infiltrating Tregs show coexpression of these markers and are therefore considered as thymus derived, recent reports call the status of Helios and Nrp1 into question (36). Helios expression was reported to reflect recent TCR stimulation in addition to thymic origin (37), and Nrp1 was shown to be lacking on a proportion of thymic Tregs (38). Alternatively, increased expression of these markers by intragraft Tregs could additionally reflect the generation of peripheral Tregs via TCR stimulation under tolerogenic conditions. We hypothesize that thymic Tregs are recruited to the graft at the beginning of an alloresponse mediated by the remaining pool of donor-reactive $\mathrm{T}$ cells (39). These graft-infiltrating Tregs are suggested to inhibit terminal differentiation (in graft-draining lymph nodes) and induce peripheral Tregs directly in the graft as shown by others (35).

In conclusion, these data provide evidence that combining Treg therapy with donor BMT induces donor-specific tolerance that is more complete than the mainly deletional tolerance induced by nonmyeloablative preconditioning (17). Tolerance achieved by therapeutic Treg treatment relies on intragraft regulatory mechanisms in which thymic Tregs continuously suppress intragraft conventional $\mathrm{T}$ cells directed toward tissue-specific donor antigens in an infectious tolerance-like manner. Our data clearly indicate that Treg treatment is preferable over nonmyeloablative cytotoxic recipient conditioning, for both safety and efficacy reasons. Treg-based but not nonmyeloablative irradiation-based protocols avoid the phenomenon of split tolerance in "stringent organs/tissues" (11) and achieve tolerance to tissue-specific donor antigens. Although others aimed to determine intragraft immunomodulatory mechanisms in mixed chimerism (40) or peripheral tolerance models (41), this is the first study employing a clinically relevant fully MHC- and minor antigen-mismatched strain combination. The findings presented herein should be taken into consideration when developing tolerance protocols for clinical use.

\section{Methods}

Animals. Female C57BL/6 (B6, recipient, H-2 ${ }^{\mathrm{b}}$ ), BALB/c (donor, $\left.\mathrm{H}-2^{\mathrm{d}}\right)$, and $\mathrm{C} 3 \mathrm{H} / \mathrm{HeNCrl}(\mathrm{C} 3 \mathrm{H}$, third party, $\mathrm{H}-2^{\mathrm{k}}$ ) mice were purchased from Charles River Laboratories. Female $\mathrm{RAG}^{-/-}$(B6 background), B6.SJL-PtprcaPep3b/BoyJ (B6/CD45.1, congeneic, H-2 ${ }^{b}$ ), and B10.D2 (H-2 ${ }^{\mathrm{d}}$ ) were purchased from Jackson Laboratories. Female BALB/c-Tg(Phlp5-GFP)304Biat (BALB/c-Phlp5, ubiquitous expression of Phlp5 antigen on BALB/c background) mice have been developed in our lab (42) and were bred under specific pathogen-free (SPF) conditions. Mice were housed under SPF conditions in individually ventilated filter cages (up to 5 mice) on sterile standard bedding; sterile water and a standard pellet diet were given ad libitum. Housing rooms were equipped with a 12 -hour light cycle. Mice were used at $6-8$ weeks of age with an average weight of $18-20 \mathrm{~g}$.

$B M T$ protocols. Groups of age-matched B6 recipients received approximately $2 \times 10^{7}$ unseparated $\mathrm{BM}$ cells recovered from BALB/c donors (i.v.; d0) and costimulation blockade with anti-CD154 mAb (MR1; $1 \mathrm{mg}, \mathrm{d}$, BioXCell) and CTLA4Ig (Abatacept; $0.5 \mathrm{mg}, \mathrm{d} 2$, BMS Pharmaceuticals). Groups of mice received additional Treg treatment $\left(1 \times 10^{6}\right.$ in vitro-activated natural Tregs [nTregs] i.v.; d0; ref. 43) and a short course of rapamycin ( $0.1 \mathrm{mg} /$ mouse, $\mathrm{d}-1 / 0 / 2$, LC laboratories) (15). Alternatively, BMT recipients received 3 Gy or 9 Gy TBI prior to BMT (d-1) using a Xylon x-ray (44). Groups of mice received anti-CD25 (PC61; $0.5 \mathrm{mg} \mathrm{d0}, 0.35 \mathrm{mg} \mathrm{d} 3 / 7 / 10$ ), anti-CTLA4 (9H10; $1 \mathrm{mg} \mathrm{d0}, 0.5 \mathrm{mg} \mathrm{d} 2 / 4 / 6$ ), or anti-PD1 (J43; $0.5 \mathrm{mg} \mathrm{d} 0,0.25 \mathrm{mg} \mathrm{d} 2 / 4 / 6 / 8$ ) (all BioXcell) late after BMT or skin grafting if indicated. Antibodies were injected i.p. in a volume of $500 \mu 1$.

Generation of $C D 4^{+} C D 25^{+}$Tregs. Tregs were generated as described previously (15). Briefly, cells were isolated from spleen and lymph nodes of naive B6 mice and purified by magnetic bead separation $\left(\mathrm{CD} 4^{+} \mathrm{CD} 25^{+}\right.$ Regulatory T cell Isolation Kit, Miltenyi Biotec). Purity of separated cells was greater than 90\%. Cells were used in vivo after cultivation for 5 days in plates coated with $10 \mu \mathrm{g} / \mathrm{ml}$ anti-CD3 (145-2C11) and $1 \mu \mathrm{g} / \mathrm{ml}$ anti-CD28 (37.51) (both BD Pharmingen) in the presence of $100 \mathrm{U} / \mathrm{ml} \mathrm{IL}-2$ (Sigma-Aldrich).

Murine skin and heart transplantation. Full-thickness tail skin was grafted 4-6 weeks after BMT and visually inspected thereafter at short intervals. Grafts were considered to be rejected when less than $10 \%$ 
remained viable. Cervical heterotopic heart transplantation was performed 6-8 weeks after BMT with a modified cuff technique for revascularization as described previously (17). Briefly, after preparation of the right external jugular vein and common carotid artery cuffs, the donor heart was harvested and transferred to the neck of the recipient. Main pulmonary artery and aorta were anastomosed to the recipient's external jugular vein and carotid artery, respectively. Cardiac allograft survival was determined by daily palpation and observation under the microscope, with complete cessation of heart beats indicating end of graft survival (confirmed by histopathological analysis).

Anti-donor antibodies. Recipient serum for FCXM was harvested more than 3 weeks after skin/cardiac transplantation, and at the end of followup, the serum was heat-inactivated and incubated with recipienttype (B6), donor-type (BALB/c or BALB/c-Phlp5), and third-party-type $(\mathrm{C} 3 \mathrm{H})$ thymocytes. Binding of serum IgG Abs to thymocytes was analyzed by flow cytometry using FITC-conjugated rat anti-mouse IgG1 and IgG2a/2b (BD Pharmingen). FCXM, as generally used in such experiments, would detect mainly humoral response against $\mathrm{MHC}$ class I.

Antibodies and FCM analysis. Multicolor FCM analysis of Treg phenotype, multilineage chimerism, and $\mathrm{V} \beta$-subunit expression (TCR V $\beta 5.1 / 5.2$ and V $\beta 11$ surrogate parameter for donor-reactive $\mathrm{T}$ cell clones and V $\beta 8.1 / 8.2$ as nonspecific control) was performed as described previously in peripheral blood, spleen, and thymus. Chimerism was calculated as the net percentage of donor MHC class $\mathrm{I}^{+}\left(\mathrm{H}-2 \mathrm{D}^{\mathrm{d}}, 34-2-12\right)$ cells among leukocyte lineages over time in peripheral blood (CD4, CD8, CD19, CD11b/Mac1) and in thymus (CD4, CD8, CD11b/Mac1, CD11c), BM (CD19, CD11b/Mac1), and spleen (CD4, CD8, CD19, $\mathrm{Nk1.1)}$ at the time of sacrifice (15). Mice were considered chimeric if donor cells were detectable by flow cytometry within both the myeloid lineage and at least one lymphoid lineage. For Treg analysis, mAbs with specificity against CD4 (RM4-4), CD25 (7D4), and CD62L (L-selectin, Mel-14) were used. Phenotype analysis further included mAbs with specificity against CD45, CD3, GITR, CTLA4, PD-1, CD44 (all BioLegend), and Nrp1 (R\&D Systems). For intracellular staining, an intracellular staining kit (eBioscience) was used according to the manufacturer's protocol followed by incubation with FoxP3 (FJK-16s, eBioscience), Helios (22F6), and Ki67 (SolA15, eBioscience), respectively. Fc blockade with purified CD16/CD32 (2.4G2, BD Biosciences) prior to surface staining (or after fixation/permeabilization) was used to minimize unspecific binding. Antibodies were obtained from BioLegend unless noted otherwise. Flow cytometric analysis was performed with BD FACS CantoII and FlowJo software.

Histologic analysis. Sections $(4-\mu \mathrm{m})$ were cut from paraffin-embedded tissue fixed in $4.5 \%$ formalin (with a buffered $\mathrm{pH}$ of 7.5), stained with H\&E or Giemsa and Elastica van Gieson (EvG) stain according to standard protocols, and analyzed by an experienced clinical pathologist in blinded fashion. Skin allografts were scored according to Banff 2007 working classification of skin-containing composite tissue allograft pathology (45). Heart allografts were scored according to the International Society for Heart and Lung Transplantation (ISHLT) 2005 Guidelines for cellular rejection (46).

For immunofluorescent analysis, $6-\mu \mathrm{m}$ frozen sections of thymus were prepared and subsequently fixed with precooled acetone. Unspecific binding was blocked with 1\% BSA, 10\% goat serum, and 0.1\% Tween 20 diluted in Tris-buffered saline ( $\mathrm{pH}$ 7.6). CD11c was stained with Alexa Fluor 647 anti-mouse CD11c antibodies (clone N418, BioLegend), and donor MHC II (I-E ${ }^{\mathrm{k} / \mathrm{d}}$ ) was stained with Alexa Fluor 488 antimouse I-Ek/rat RT1D antibodies (clone 14-4-4S, BioLegend). Stained sections were analyzed on a Nikon Eclipse Ti-E epifluorescence microscope using the imaging software NIS Elements BR (Nikon).

Statistics. A 2-sided Student's $t$ test with unequal variances was used to compare percentages of V $\beta$ family ${ }^{+}$cells, chimerism levels, and phenotypical markers between groups. Fisher's exact test was used to compare chimerism rates between groups and rejection scores. Skin and heart allograft survival were calculated according to the Kaplan-Meier product limit method and compared between groups using the log-rank test. A $P$ value less than 0.05 was considered to be statistically significant. Data are presented as mean $\pm \mathrm{SD}$ in all figure parts containing error bars.

Study approval. All experiments were discussed and approved by the Ethics and Animal Welfare Committee of the Medical University of Vienna and were performed in strict accordance with national and international guidelines of laboratory animal care. All animals received humane care in compliance with FELASA and ARRIVE and are covered by ethics vote of the Austrian Federal Ministry of Science, Research and Economy BMWKF-66.009/0195-II/10b/2009 and GZ 66.009/0230- II/3b/2011. All surgeries were performed under general anesthesia employing a mixture of ketamine $(100 \mathrm{mg} / \mathrm{kg})$ and Xylazine $(5 \mathrm{mg} / \mathrm{kg}$ ) i.p. The concept of 3 Rs (replacement, refinement, and reduction) were implemented in 
study design of the approved ethical protocol. All efforts were made to minimize distress and group size. The number of mice in each specific group is provided in the figure legend.

\section{Author contributions}

NP designed and performed experiments, analyzed data, and wrote the manuscript; BM, LU, KH, CS, $\mathrm{AMF}$, and UB conducted experiments and acquired data; FW acquired and analyzed data; TW designed research and wrote the manuscript. All authors approved the final version of the manuscript.

\section{Acknowledgments}

We thank Megan Sykes for helpful discussions and Kylie Webster and Jonathan Sprent for critical reading of the manuscript. This work was supported by the Austrian Science Fund (FWF; TRP151-B19 and Doctoral Programme W1212 to TW) and the Else Kroener-Fresenius-Stiftung (EFKS to NP).

Address correspondence to: Thomas Wekerle, Department of Surgery, Vienna General Hospital, Waehringer Guertel 18; 1090 Vienna, Austria. Phone: 43.1.40400.39380; Fax: 43.1.40400.39382; E-mail: Thomas. Wekerle@meduniwien.ac.at.

1. Kawai T, et al. Long-term results in recipients of combined HLA-mismatched kidney and bone marrow transplantation without maintenance immunosuppression. Am J Transplant. 2014;14(7):1599-1611.

2. Scandling JD, et al. Chimerism, graft survival, and withdrawal of immunosuppressive drugs in HLA matched and mismatched patients after living donor kidney and hematopoietic cell transplantation. Am J Transplant. 2015;15(3):695-704.

3. Leventhal J, et al. Tolerance induction in HLA disparate living donor kidney transplantation by donor stem cell infusion: durable chimerism predicts outcome. Transplantation. 2013;95(1):169-176.

4. Granados JM, Benichou G, Kawai T. Hematopoietic stem cell infusion/transplantation for induction of allograft tolerance. Curr Opin Organ Transplant. 2015;20(1):49-56.

5. Elias N, Cosimi AB, Kawai T. Clinical trials for induction of renal allograft tolerance. Curr Opin Organ Transplant. 2015;20(4):406-411.

6. Sykes M. Mixed chimerism and transplant tolerance. Immunity. 2001;14(4):417-424.

7. Boehmer H, Sprent J, Nabholz M. Tolerance to histocompatibility determinants in tetraparental bone marrow chimeras. $J$ Exp Med. 1975;141(2):322-34.

8. Wells $\mathrm{AD}$, et al. Requirement for T-cell apoptosis in the induction of peripheral transplantation tolerance. Nat Med. 1999;5(11):1303-1307.

9. Li Y, Li XC, Zheng XX, Wells AD, Turka LA, Strom TB. Blocking both signal 1 and signal 2 of T-cell activation prevents apoptosis of alloreactive T cells and induction of peripheral allograft tolerance. Nat Med. 1999;5(11):1298-1302.

10. Sachs DH, Kawai T, Sykes M. Induction of tolerance through mixed chimerism. Cold Spring Harb Perspect Med. 2014;4(1):a015529.

11. Madariaga ML, Kreisel D, Madsen JC. Organ-specific differences in achieving tolerance. Curr Opin Organ Transplant. $2015 ; 20(4): 392-399$

12. Riley JL, June CH, Blazar BR. Human T regulatory cell therapy: Take a billion or so and call me in the morning. Immunity. 2009;30(5):656-665.

13. Tsang JY, et al. Conferring indirect allospecificity on $\mathrm{CD} 4^{+} \mathrm{CD} 25^{+}$Tregs by TCR gene transfer favors transplantation tolerance in mice. J Clin Invest. 2008;118(11):3619-3628.

14. Joffre O, Set al. Prevention of acute and chronic allograft rejection with $\mathrm{CD} 4^{+} \mathrm{CD} 25^{+} \mathrm{Foxp} 3^{+}$regulatory T lymphocytes. Nat Med. 2008;14(1):88-92.

15. Pilat N, et al. Treg-therapy allows mixed chimerism and transplantation tolerance without cytoreductive conditioning. Am $J$ Transplant. 2010;10(4):751-762.

16. Wekerle T, et al. Extrathymic T cell deletion and allogeneic stem cell engraftment induced with costimulatory blockade is followed by central T cell tolerance. J Exp Med. 1998;187(12):2037-44.

17. Pilat N, et al. T-regulatory cell treatment prevents chronic rejection of heart allografts in a murine mixed chimerism model. J Heart Lung Transplant. 2014;33(4):429-437.

18. Pilat N, et al. Therapeutic efficacy of polyclonal tregs does not require rapamycin in a low-dose irradiation bone marrow transplantation model. Transplantation. 2011;92(3):280-288.

19. Anderson MS, et al. Projection of an immunological self shadow within the thymus by the aire protein. Science. 2002;298(5597):1395-1401.

20. Wing K, et al. CTLA-4 control over Foxp3+ regulatory T cell function. Science. 2008;322(5899):271-275.

21. Francisco LM, et al. PD-L1 regulates the development, maintenance, and function of induced regulatory T cells. J Exp Med. 2009;206(13):3015-3029.

22. Bigenzahn S, et al. The role of non-deletional tolerance mechanisms in a murine model of mixed chimerism with costimulation blockade. Am J Transplant. 2005;5(6):1237-1247.

23. Pasquet L, Douet JY, Sparwasser T, Romagnoli P, van Meerwijk JP. Long-term prevention of chronic allograft rejection by regulatory T-cell immunotherapy involves host Foxp3-expressing T cells. Blood. 2013;121(21):4303-4310.

24. Keir ME, et al. Tissue expression of PD-L1 mediates peripheral T cell tolerance. J Exp Med. 2006;203(4):883-895. 
25. Mueller DL. Mechanisms maintaining peripheral tolerance. Nat Immunol. 2010;11(1):21-27.

26. Kendal AR, Waldmann H. Infectious tolerance: therapeutic potential. Curr Opin Immunol. 2010;22(5):560-565.

27. Walker LS, Chodos A, Eggena M, Dooms H, Abbas AK. Antigen-dependent proliferation of CD4 $4^{+} \mathrm{CD} 25^{+}$regulatory T cells in vivo. J Exp Med. 2003;198(2):249-258.

28. Lin X, et al. Advances in distinguishing natural from induced Foxp3(+) regulatory T cells. Int J Clin Exp Pathol. 2013;6(2):116-123.

29. Scadden DT. The stem-cell niche as an entity of action. Nature. 2006;441(7097):1075-1079.

30. Al-Adra DP, Anderson CC. Mixed chimerism and split tolerance: mechanisms and clinical correlations. Chimerism. 2011;2(4):89-101.

31. Legoux FP, et al. CD4(+) T cell tolerance to tissue-restricted self antigens is mediated by antigen-specific regulatory $\mathrm{T}$ cells rather than deletion. Immunity. 2015;43(5):896-908.

32. Zinkernagel RM, Althage A, Callahan G, Welsh RM Jr. On the immunocompetence of H-2 incompatible irradiation bone marrow chimeras. J Immunol. 1980;124(5):2356-2365.

33. Thornton AM, et al. Expression of Helios, an Ikaros transcription factor family member, differentiates thymic-derived from peripherally induced Foxp3 ${ }^{+}$T regulatory cells. J Immunol. 2010;184(7):3433-3441.

34. Weiss JM, et al. Neuropilin 1 is expressed on thymus-derived natural regulatory $\mathrm{T}$ cells, but not mucosa-generated induced Foxp3 $3^{+}$T reg cells. J Exp Med. 2012;209(10):1723-1742.

35. Shevach EM, Thornton AM. tTregs, pTregs, and iTregs: similarities and differences. Immunol Rev. 2014;259(1):88-102.

36. Szurek E, et al. Differences in expression level of Helios and Neuropilin-1 do not distinguish thymus-derived from extrathymically-induced $\mathrm{CD}^{+}{ }^{+} \mathrm{Foxp}^{+}$regulatory T cells. PLoS One. 2015;10(10):e0141161.

37. Gottschalk RA, Corse E, Allison JP. Expression of Helios in peripherally induced Foxp3 ${ }^{+}$regulatory T cells. J Immunol. 2012;188(3):976-980.

38. Singh K, Hjort M, Thorvaldson L, Sandler S. Concomitant analysis of Helios and Neuropilin-1 as a marker to detect thymic derived regulatory $\mathrm{T}$ cells in naive mice. Sci Rep. 2015;5:7767.

39. Boer K, et al. Thymus-derived regulatory T cells infiltrate the cardiac allograft before rejection. Transplantation. 2015;99(9):1839-1846

40. Domenig $\mathrm{C}$, et al. Roles of deletion and regulation in creating mixed chimerism and allograft tolerance using a nonlymphoablative irradiation-free protocol. J Immunol. 2005;175(1):51-60.

41. Graca L, Cobbold SP, Waldmann H. Identification of regulatory T cells in tolerated allografts. J Exp Med. 2002;195(12):1641-1646.

42. Baranyi U, et al. Cell therapy for prophylactic tolerance in IgE-mediated allergy [published online ahead of print March 31, 2016]. EBioMedicine. doi:10.1016/j.ebiom.2016.03.028.

43. Pilat N, et al. Polyclonal recipient nTregs are superior to donor or third-party Tregs in the induction of transplantation tolerance J Immunol Res. 2015;2015:562935.

44. Kuess P, Bozsaky E, Hopfgartner J, Seifritz G, Dorr W, Georg D. Dosimetric challenges of small animal irradiation with a commercial X-ray unit. Z Med Phys. 2014;24(4):363-372.

45. Cendales LC, et al. The Banff 2007 working classification of skin-containing composite tissue allograft pathology. Am J Transplant. 2008;8(7):1396-1400.

46. Stewart S, et al. Revision of the 1990 working formulation for the standardization of nomenclature in the diagnosis of heart rejection. J Heart Lung Transplant. 2005;24(11):1710-1720. 